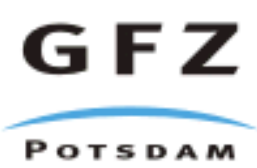

Originally published as:

Hamoudi, M., Thébault, E., Lesur, V., Mandea, M. (2007): GeoForschungsZentrum Anomaly Magnetic Map (GAMMA): A candidate model for the World Digital Magnetic Anomaly Map. - Geochemistry Geophysics Geosystems (G3), 8, Q06023

DOI: 10.1029/2007GC001638. 


\title{
GeoForschungsZentrum Anomaly Magnetic MAp (GAMMA): A candidate model for the World Digital Magnetic Anomaly Map
}

\author{
M. Hamoudi, E. Thébault, V. Lesur, and M. Mandea
}

\begin{abstract}
The World Digital Magnetic Anomaly Map (WDMAM) is an ongoing effort towards the mapping of worldwide available aeromagnetic data. It is led by a task force of the International Association for Geomagnetism and Aeronomy (IAGA) and aims at distributing a global map in printed and digital forms. In this paper, we describe in details our candidate model which has to be evaluated by the IAGA task force together with five other candidate maps. After discussing the quality of the available data, we apply a simple but effective method to successfully process, reduce and merge together individual compilations. The near surface data are corrected using global field models and further refined with a 2D polynomial corrections. After the upward continuation to $5 \mathrm{~km}$ altitude, data are re-sampled to a 3 minutes grid and merged together. We then calculate a spherical harmonic model up to degree 199 and analyze the magnetic spectrum of the global map. This helps us to confirm that wavelengths larger than $400 \mathrm{~km}$ are spurious at a global scale in aeromagnetic compilations. Therefore, we substitute them using a satellite based lithospheric field model (MF5) to degree 100.
\end{abstract}




\section{Introduction}

The importance of aeromagnetic and marine magnetic surveys to understand the geology has been long demonstrated, but a number of problems remain difficult to solve when considering regional compilations only. A worldwide magnetic anomaly model derived from the merging of satellite, airborne, marine and land magnetic data can provide a comprehensive view of continentalscale magnetic trends, not available in individual data sets. It also helps linking widely separated areas of outcrop, unifies disparate tectonic and geological studies (Reeves and De Witt, 2000, for instance). Such a global anomaly map will thus be a powerful tool for further evaluation of the lithospheric structure, geologic processes and tectonic evolution of continental or oceanic areas (Vine, 1966). These studies require consistent data sets over thousands kilometres distances spanning national boundaries.

The World Digital Magnetic Anomaly Map (WDMAM) working group of IAGA aims at producing a compiled magnetic anomaly map containing all possible wavelengths useful for geological and tectonic mapping of the crust. The work presented here is a candidate model towards the final WDMAM product. The data sets used in this study were kindly provided by various organizations to the WDMAM committee (see Table 1). These magnetic compilations result from the merging of many independent regional magnetic surveys with various characteristics. Data were recorded at different epochs and altitudes, often without proper secular variation or altitude corrections. The existing final compilations have therefore errors causing significant differences between adjacent panels. These errors are clearly noticeable along the 
edges of adjacent surveys where levelling errors dominate. As a result, long wavelengths in these surveys are partly spurious and individual compilations do not easily merge.

Large compilations, such as Arctic or the North America grid, extending at scales of several thousands of kilometres are available but, so far, the challenge to handle the number of grids and specifications greatly hampered the attempt to generate a global view of magnetic anomalies. Moreover, most data are still not available for various reasons. Nevertheless, thanks to concerted and persistent efforts during last years, a large number of nearsurface magnetic grids are now available and this allows the release of the first magnetic anomaly map.

In a first section, we present the specification of each datasets, the coordinate systems used and, when available, the original main field reduction and the overall statistics. We also report on prominent observed inconsistencies. These discussions help us to define a grid precedence order according to their estimated quality in section 2 . In section 3 , we apply a simple and effective method to merge the individual grids. We correct the large wavelengths by iteratively adjusting a low-degree main field for different epochs until minimum mean anomaly intensity is obtained. We adjust the grid by removing a regional polynomial to the compilation in order to improve the statistical characteristics of each data distribution. Following the recommendation of the WDMAM committee, data are upward continued to 5km above the World Geodetic System 1984 (WGS84) reference ellipsoid and gridded on a 3'x3' grid (about $5 \mathrm{~km}$ spacing). We then apply dedicated software in order to knit a compilation at a global scale. After a brief review of different available satellite lithospheric field models, we finally apply a global spherical harmonic filter to remove the non-physical data points, the remaining large wavelength discontinuities and the last 
inconsistencies. Wavelengths larger than spherical harmonic degree 100 , corresponding to $400 \mathrm{~km}$ maximum resolution, are removed and the currently best CHAMP anomaly field model available is subsequently added to the grid at $5 \mathrm{~km}$ altitude.

\section{Data sets}

We use the data provided to us by the WDMAM committee. We consider the compilations summarized in Table 1. Some of them are partially redundant and we discuss below how we deal with the overlapping areas.

The overall coverage is especially sparse over oceans, but also over Africa and South America where data exist without being freely accessible. The available data density greatly varies between the Northern and the Southern hemisphere and according to regional characteristics. The data quality over each region is hard to estimate as very few compilations have complete metadata information (see Table 1). When available, metadata information shows compilations to be in different coordinate systems and projections. All compilations result from the stitching together of smaller surveys carried out at various altitudes and the individual panels were, or were not, upward continued to a common altitude. For some compilations, these information are provided but since in general the mean altitude, or the mean terrain clearance with respect to the mean sea level, is not systematically known, we have no other choice but to upward continue the data in the latest stage of the final compilation.

Panels inside each individual compilation were derived for different epochs and reduced with either local polynomials or IGRF/DGRF models. In most cases, it is difficult to find out which model was used to reduce the data. The final patch-worked grids are thus 
prone to mismatch in anomaly shapes and strengths that may easily be confused with magnetic anomalies. The lack of absolute reference makes it difficult to restore the large wavelengths. Data sampling is also not homogeneous. It varies from about $50 \mathrm{~km}$ and $30 \mathrm{~km}$ for respectively India and both Africa and South America grids, to $1 \mathrm{~km}$ spacing for North America or Australia, for instance. Determining the grid resolution for each compilation would require a full spectral analysis that was not performed here. Checking the consistency between two overlapping grids is therefore challenging in some areas where the actual resolution is not known. Moreover, the resolution is usually not homogeneous within the compilations themselves and some regions artificially appear devoid of small magnetic anomalies. In future editions of the WDMAM, this problem should be identified before any interpretation is carried out.

Three datasets are used for cosmetic reasons until better grids are provided: part of Africa and South America in the Southern hemisphere and the north west of Indian grid constructed from ground stations.

Several versions exist for some compilations. For instance, version 4 of Australian and adjacent marine areas data were considered. In general, redundant panels were simply removed from the final dataset if they did not bring resolution improvement. Hence, Mexico grid was removed as the data were included in the North American compilation. Japan grid was part of the East Asia compilation and was not considered. To the contrary, Fennoscandia and Austria, included in the Arctic and European compilation, have a better resolution. After a thorough analysis, project Magnet dataset was removed in order to minimize the associated spurious effects over North America and Australia. Some compilations such 
as China or Mongolia were obtained from digitization of shaded colour maps and thus discarded. In the latter cases, the quality of the grid could not be objectively testified but visual inspections and statistics show discontinuities, noise, unrealistic linear features spreading over thousands of kilometres and obvious edge effects.

Regarding all these aspects, although some grids have interesting characteristics, only a few files like the French, Italian and Spanish grid, for instance, posses the complete information to fully control the data processing. The French grid is also derived from a oneyear survey carried out at a nearly constant altitude and reduced to $3 \mathrm{~km}$ altitude above mean sea level. Line levelling, main field and external field corrections using the nearest observatory were performed (Le Mouël, 1969). The grid also comes with the total field intensity and, as it slightly overlaps with the European compilation, we use the French dataset to level the European compilation near the French boundary. Similarly, the Italian compilation is a corrected grid provided with the regional polynomial used to reduce the total intensity (Chiappini et al., 2000). It is thus possible to further correct for a global core field model or a given epoch. Information on the core field reduction is not provided for the Australian compilation, it nonetheless provides high quality data that are consistent over large scale with satellite observations.

Before applying filtering and correction procedures, the data not provided in a geographic coordinate system are converted to the global WGS84 reference ellipsoid using transformation formula (Snyder, 1972) and a dedicated software (Oasis Montaj, GeoSoft ${ }^{\odot}$ ).

\section{Grid inconsistencies and discontinuities}


Some problems discussed above, inherent in each grid, are not directly noticeable but appear simply by displaying the grids on the sphere. Discontinuities are a major issue visible on all compilation edges. It is clearly noticeable at the Northern border between the American compilation and the Arctic compilation, for instance. Regarding the specificities and the varieties of survey composing the American NAMAG compilation, and despite the preliminary CM4 model reduction (Ravat et al., 2003) we expect a poor resolution for wavelengths greater than $200 \mathrm{~km}$. The most evident discontinuities are between continental and oceanic compilations. The marine track-lines data suffer from data reduction, line levelling and instrumental biases that require a full reprocessing not performed here. The Canary grid has unrealistic magnitudes that were adjusted using adjacent compilations.

A quick inspection of basic statistics provides further details about the reliability of each dataset. The statistics help us to define a precedence order that is later used for merging grids. For some compilations, as shown in Table 2, the average anomaly intensity greatly deviates from zero. If in a first approximation we assume a dominating crustal field for wavelengths smaller than $3000 \mathrm{~km}$ (i.e. spherical harmonic degree 15), the intensity anomaly should average to zero over large distances. In that respect, the Russian and the South Asia compilations show peculiar statistics with large anomaly intensity means. This reveals either a poor core field reduction or spurious long wavelengths. The European compilation has also a comparatively large mean $(\sim 16 \mathrm{nT})$, which leads to large discontinuities with all surrounding compilations. Nevertheless, the metadata information indicate that the European compilation was purposefully reduced with the DGRF1980 and the core field contributions for spherical harmonic degrees 11-15 explain mostly this relatively high mean. 
The standard deviation is usually between 100nT and 200nT over continents, but the marine data shows a standard deviation reaching 930nT. This suggests the persistence of noise, bad tracks or outliers. For this reason, the correlation between marine and satellite data is particularly poor. One of the reasons is that marine data are not corrected for external or daily magnetic variations. In addition, the crossover tracks, recorded at different times over long periods, are naturally contaminated by the magnetic secular changes and external fields and large mismatches are observed.

The calculation of the arithmetic sum is informative and shows how well the residuals distribute around the mean. For a pure anomaly field, we expect a Gaussian-like distribution with zero mean. A non symmetric anomaly distribution around the mean possibly indicates some positive or negative outliers tailing on the distribution. The marine compilation has the larger arithmetic sum closely followed by the Russian, the Australian, and the South Asia compilations. Note that the comparatively high mean for the European compilation does not imply a particularly high arithmetic mean, showing evidence that the European grid does not contain prominent outliers.

\section{Partial correction and adjustments}

This section introduces the pre-processing designed to improve the compatibility in the overlapping areas. We did not systematically analyse the grid consistencies at regional scales except for few oceanic compilations. Most compilations were obtained by combining, locating and processing individual survey to generate a wider compilation. We assumed rather reliable and uniform grid qualities, unless otherwise stated as for the European grid. 


\section{4.a.Visual inspection}

A rough technique to smooth the inconsistencies is to bin data on a coarser grid. Here, we keep the original grid as far as possible in our processing and we try to identify the evident wrong isolated points or track lines.

For the oceanic compilation, we identified bad tracks spreading over thousands of kilometres in the Pacific Ocean and we record a maximum anomaly field up to $45931.7 \mathrm{nT}$. We manually removed these tracks. We also removed track lines crossing the African continents showing possible instrumental deficiencies and/or location problems in the oceanic data. Off shore Senegal, a few cross-shaped anomalies were removed.

In the Getech South American and the African compilations the original data have been decimated. The resulting grids have a poor quality and a low resolution. The Bangui anomaly, for instance, has a rather unusual shape and it is sometimes difficult to delineate clearly other well-known anomalies.

The Indian grid based on repeat stations was considerably reduced and we kept only data following the magnetic map boundaries published in Qureshy (1982) on the eastern coast.

The European grid as been shifted by few kilometres such that its anomaly field coincide with known total intensity anomalies over Germany. This grid has been used only when no other data set was available over an area.

At last, most of the grids were prone to edge effects possibly caused by either remaining large wavelengths or a Fourier filtering over large distances that was performed at the latest stage of their compilation process. We could not properly identify and correct for these effects but some anomalies have unusual shapes and are shifted. Europe and Russia were bounded with dataset having 
complete metadata information. We were thus able to better constraint the merging between adjacent grids in these regions.

\section{4.b.Global field correction}

Compilations were formerly reduced with different IGRF/DGRF core field models sometimes followed by 2D polynomial fitting or a Cartesian Fourier filtering. For a few grids over the European continent, we have the correct regional polynomial parameters (or the DGRF model) and sometimes even the total intensity. It was thus easy to add the exact core field model and to remove the CM4 (Comprehensive model, Sabaka et al., 2004) for the same epoch. For the other compilations, we tried to restore the closest core field model by iteratively looking for the lowest residuals at different epochs after adding a DGRF model (spherical degree 10) and subtracting the CM4 model to degree 15 . The complete period between 1960 and 2002 was spanned. The Russian compilation seems to be outside the CM4 time span and the procedure was not applied. Figure 1 shows a few examples and results at this step. The residuals have no particular shape but a minimum can be found (although sometimes not exactly unique). Since we have no information about the removed model, this ad hoc procedure may be arguable. Nevertheless, this step improves the data statistics presented in Table 3 by lowering the mean intensity anomaly towards zero. When considering the total field intensity data, the minimum is obvious. For example, the curve for France shows that the variation of the residual over the 40 years interval is of the magnitude order of the secular variation. The same result is obtained for Italy or Spain. This procedure is thus a way to better correct for the secular variation between the different compilations. Since original data may contain various artefacts caused by the different model biases, it is also a mean to reduce 
the data with the same core field model. In general, the continuity in overlapping areas between adjacent compilations was improved.

\section{4.c.Regional polynomial correction}

In order to further improve the statistics, a regional second order polynomial is removed from all grids in the WGS84 geographic reference so that we avoid Cartesian distortion due to the Earth's curvature. This was not possible over geographic poles and Arctic and Antarctic compilations were processed using Cartesian reference frame instead. The polynomial fitting was not applied to the Australian compilation where we assumed that the long wavelengths were valid. Removing a polynomial carries the risk of destroying all possible correlation between ground and satellite data as is illustrated below. The spectrum is modified as the polynomial correction is a function of the grid size but wavelengths larger than spherical harmonic degree 100 are ultimately filtered out (see subsection 4.f). After this correction, the grids have arguably the correct properties characterising anomaly fields; the average anomaly intensity and the arithmetic sum are almost zero (Table 3) and the residual histograms resemble a Gaussian distribution (see Figure 2).

\section{4.d.Upward continuation}

The original grids are provided at various but generally constant altitudes within the same dataset (see Table 1). This may be untrue for some compilations but this problem could not be addressed here as correct altitudes cannot be recovered. We applied the filter at the specified mean altitude. Oceanic datasets were also upward continued in order to avoid a too sharp transition between oceancontinent boundaries. 
The grids smaller than 2000km width, like Argentina, Austria, Fennoscandia, Italy, France, Mexico and Spain were directly upward continued to $5 \mathrm{~km}$ altitude above the geoid. Larger compilations were split into $2000 \mathrm{~km}$ by $2000 \mathrm{~km}$ squares, which were upward continued individually. This dimension corresponds to the maximum size for which the Earth's curvature can be neglected (Nakagawa et Yukutake, 1985). This requires a Nyquist re-sampling of the original grid on a $2.5 \mathrm{~km}$ regular grid in a Cartesian reference frame so that a maximum resolution of $5 \mathrm{~km}$ is obtained. The spacing is chosen so that we have the best trade-off between speed and efficiency. This high re-sampling is probably unnecessary as none of the data sets has a true $5 \mathrm{~km}$ resolution. The upward continued data are then calculated back to the original geographic data locations. The GeoSoft $\odot$ algorithm includes a detrending of the data and works in the Fourier domain. Each panel slightly overlap with the adjacent ones and we systematically check the consistency of the result over the overlapping areas. We cannot report on noticeable distortion, and the overall statistics in Table 3 are nearly preserved. It is worth noting that after the upward continuation the edge effects between adjacent grids were not significantly but slightly enhanced.

\section{4.e.Merging the grids}

The merging process does not rely on physical assumptions and whether merging the neighbouring grids or not is a matter of choice. We first considered the complete overlap between different grids in order to obtain a final grid averaging the full available information. It occurred to us that this procedure was generating spurious long and intermediate wavelengths in the merged dataset. Moreover, it gave the same weight to dataset of different qualities, which was unacceptable. As a result, no more than fifty kilometres overlap were allowed between redundant datasets. We thus 
obtained a merged grid smooth and closer to the independent original grids. Large amount of both North America and Arctic compilation data were removed. In South Africa, data were cut when overlapping SaNaBoZi data; whereas in South America, they were cut when overlapping Argentina data.

The preliminary processing from paragraph 4.a to 4.d reduces the large discontinuities but does not fully remove them. We use the grid-knitting tool of $\mathrm{GeoSoft}^{\odot}$ in order to smooth out the transition between adjacent grids. Some spurious wavelengths are created that will be mostly filtered out at the last stage of the processing. We were especially careful when choosing the precedence grid order. We already argued that French, Italian, and European grids were easier to process as we have the necessary metadata information to better control the processing. Merging large compilations with small compilations includes a risk, because the adjustment is better constraint by the large compilation even if the small one has apparently a better quality. Before merging the grids, we generate compilations with comparable grid sizes.

The precedence order is as follows: French, Italian and Spanish grids are first merged together. We then built a second grid from the Finland, Fennoscandian, European and Austrian grids. This two new compilations were merged together and the grid from Russia, Eurasia, Middle East, Antarctica and North America were successively added to the compilation. The remaining grids were not merged as they did not have overlap with the grids listed above.

At this stage, a 3'x3' grid is generated using GMT (Wessel and Smith, 1991, version 4.1.4). Each node of our final grid is associated to an index corresponding to the data set used to calculate the anomaly field value at that node (last column of Table 1). When a grid node is associated with several data sets, the given 
index correspond to the weighted average of the data sets indices. Compilations without index in Table 1 were not used in our merging. Marine data were interpolated whenever the data density was estimated high enough, otherwise left as single track data.

\section{4.f.Final Global filtering}

Any discontinuity caused by large wavelengths introduced during the merging can be filtered out at a global scale by a spherical harmonic transformation. Compilation boundaries are still visible, especially between Arctic, Oceanic and Australian grids. In addition, the mismatch between European and Russian compilation remains. We thus perform a spherical harmonic transform and filter out wavelengths larger than spherical harmonic degree 100 in our global grid to replace them with a continuous satellite based model. Two different techniques were envisaged for filtering and smoothing the final grid. A first option consists in filtering the grid using Fourier transforms. This procedure is relatively fast but is a non-potential method. Thus, the coefficients could not be used for predicting the three components of the magnetic field. Moreover, it is difficult to remove remaining non-physical magnetic measurements. In this section, we consider the magnetic anomaly field as the projection of the crustal field onto a core field model: The grid is interpolated on the knots of the sampling theorem given in Driscoll and Healy (1994), the parameters up to spherical harmonic degree 199 were obtained by least-squares (maximum resolution of $80 \mathrm{~km}$ ). For higher degrees, the coefficients are difficult to obtain and the processing is time-consuming, as the power is very low. This is due to data gaps and heterogeneous resolution at the global scale. The anomaly field is linearized and projected on the 1990 core field, whose coefficients are extracted from the CM4 model to degree 15 (Sabaka et al., 2004). It is thus believed that the estimated Gauss coefficients represent better the 
three components of the magnetic field anomaly. Interestingly, despite our careful pre-processing, the first iteration shows evidence of remaining outliers and non-physical data points. This is characterized by the presence of strong spikes creating oscillations spreading over large distances. This carries the risk of introducing artificial anomalies at all wavelengths in our final map. Some extra points are thus removed from Argentina, Argentina coastline, near Santa Helena Island and around Madagascar. The procedure was then redone without apparent other artefacts.

Figure 3 shows that over Western Europe, the European and the French grids had an important incompatibility that was attenuated during the processing. The compatibility was improved trough the filtering and major discontinuities were smoothed out.

In Figure 4 we compare the spectrum of our spherical harmonic model with the most recent lithospheric field models, MF4, MF4x (Lesur and Maus, 2006) and MF5 (Maus et al., 2007). The nearsurface WDMAM spectrum can be divided into three parts. The first part of the spectrum, from degrees 1 to 40, is comparatively steeper, has an excess of power and is more irregular. This suggests that large wavelengths are indeed partly spurious even if the correlation analysis (Figure 5) shows a better agreement than expected ( 0.5 in average for these degrees). Degrees 41 to 89 are more stable and their variation is consistent with satellite-based spectrum. From degree 90, a small offset is noticeable whose origin remains unclear. We may venture that some recent grids were compiled using a satellite based model derived to degree 90 as a prior information. This could have induced this lack of power. Indeed, MF5 has a lack of power explained by the processing applied to satellite data in order to obtain a robust model from noisy measurements. The spherical harmonic correlation analysis (Langel and Hinze, 1998) between MF5 and our near-surface model 
(Figure 5) shows an average correlation for all degree of 0.61 but again, beyond degree 40 it is more stable and reaches 0.75 for $\mathrm{n}=91$. We notice a comparatively lack of correlation between degrees 70 and 75 that we do not explain.

\section{Substitution of long wavelengths and generation of the $3^{\prime}$ grid}

In many regions, low-orbiting satellites can map the strength and the extension of the lithospheric field. Choosing a global magnetic lithospheric field model is not a trivial task and a systematic quality analysis is required.

We discard lithospheric field models based only on satellite missions prior to the Danish Ørsted mission (1999). Langel and Hinze (1998) give a comprehensive overview of earlier satellite missions, such as MAGSAT or POGO and their outcomes.

The Danish satellite Ørsted, launched in February 1999, was followed by CHAMP (July 2000) and SAC-C (November 2000). Several models, based on these missions sharing comparable scientific instruments, were proposed in the last decade.

The comprehensive approach (CM4), initiated by Sabaka et al. (2004), uses all available data from POGO, MAGSAT, Ørsted, and only scalar data from CHAMP, as well as observatory data, in order to obtain a comprehensive model. Although the model estimates all known sources from the core to the magnetosphere, nearequatorial lithospheric field representation is probably contaminated by equatorial electrojet signature due to inclusion of dayside data. Following the same spirit, various magnetic field sources are considered by POMME 3.1 (Maus et al., 2006) using quiet time and night-side data only, which help reducing ionopsheric effects and better stabilize the model at the ground level to degree 60. Other models present a very good agreement 
with this lithospheric field (e,g, CHAOS, Olsen et al. (2006) or BGS/G/L/0706, Thomson and Lesur (2007)). These comprehensive approaches give rather robust models for the first degrees but are noisy above degree 50 .

Another approach, based on preliminary filtering, strictly focuses on the lithospheric field representation. The philosophy is to carefully select and clean the data for non-lithospheric sources. Maus et al. (2006) used this subjective approach with four years of CHAMP data to produce MF4, which is derived up to degree 90. MF4 still shows inconsistencies in the Polar Regions and Lesur and Maus (2006) managed to constrain independently Polar and Midlatitude regions. Recently, a model up to degree $100(400 \mathrm{~km}$ resolution), using only low orbital CHAMP measurements until mid2006, was released (Maus et al., 2007). It is worth noting that data filtering induces a loss of power noticeable when compared with other satellite based magnetic models but also with aeromagnetic data. Nevertheless, the MF models have degrees that are arguably suitable for large wavelengths replacement and we use MF5 to degree 100 (400km resolution). These are good compromise between resolution at the ground and smoothness of the lithospheric field at 5km altitude on the WGS84 ellipsoid. MF5 was thus added to the final grid and our resulting candidate version for the World Digital Magnetic Anomaly Map can be seen in Figure 6. Grid nodes with no aeromagnetic or marine data were filled with MF5 and their index set to 97.

\section{Conclusion}

The World Digital Magnetic Anomaly Map is a promising international effort and an ongoing project. Despite the large disparities between aeromagnetic compilations, we are able to produce a candidate model giving the essence of the worldwide 
magnetic anomaly distribution. The most important breakthrough to this project will come with the release of data in uncovered areas. At the present stage, near-surface data gaps are filled in with satellite based model that may lead to mis-interpretation in the shape and strength of the magnetic field. In the future, this problem should be addressed at all scales since data resolution is very heterogeneous even among aeromagnetic grids. Therefore, systematic interpretations should be carried out with caution and only a spectral analysis could help identifying the areas of various intrinsic resolutions. A spectral gap is thus presently unavoidable for the wavelengths from $200 \mathrm{~km}$ to $400 \mathrm{~km}$.

A major problem was to deal with oceanic data that suffer from large and numerous inconsistencies. In particular, we had to discard project Magnet data that represented too many mismatches with existing datasets. A full crossover analysis of oceanic data will be required in the future that will lead to a better line levelling and resolve the core and external field problems.

Effort continues nowadays to improve both ends of the spectrum and the upcoming Swarm mission configuration will help better resolving smaller wavelengths than degree 100. Other intrinsic problems may arise from compilations and the signal analysis processes; both could introduce artefacts and non-potential features. In addition to compilation efforts, more elaborated inverse problem techniques are being developed to merge together satellite and ground surface data at regional scales. These techniques, base on Laplace equation, may prove useful to fill the spectral gap, to merge adjacent compilations and to clean the compilation from their non-potential contributions. The latter are probably of importance as the different grid underwent resampling, decimation and successive non-potential transformations. 
In addition, applying a modelling scheme will be useful to homogenise the map resolution.

The upcoming WDMAM editions will greatly benefit from a better spatial coverage, new satellite data and improvements in data processing and modelling.

Acknowledgments - We are grateful to all organizations that kindly distributed aeromagnetic data to the WDMAM committee. $\mathrm{K}$. Hemant, Monika Korte and Yoann Lequesnel are warmly acknowledged for there efforts in acquire various datasets and for useful discussions. This work was supported by the Deutsche Forschungsgemeinshaft (DFG, SPP1097)

\section{References}

Chiappini, M., A. Meloni, E. Boschi, O. Faggioni, N. Beverini, C. Carmisciano, and I. Marson (2000), On shore - off shore integrated shaded relief magnetic anomaly map at sea level of Italy and surrounding areas, Annali di Geofisica, 43(5), 983-989.

Driscoll, J.R. and Healy, D.M. (1994), Computing Fourier transforms and convolutions on the 2-sphere. Adv. Appl. Maths. 15, 202-250.

Langel, R.A, and Hintze W.J (1998), The magnetic field of the Earth's lithosphere - The Satellite Perspective, Cambridge University Press.

Le Mouël, J. L. (1969), Sur la distribution des éléments magnétiques en France, PhD Thesis, Université de Paris, Paris. 
Lesur, V., and S. Maus (2006), A global lithospheric magnetic field model with reduced noise level in the Polar Regions, Geophys. Res. Lett., 33, L13304, doi:10.1029/2006GL025826.

Maus, S., M. Rother, C. Stolle, W. Mai, S. Choi, H. Lühr, D. Cooke, and C. Roth (2006), Third generation of the Potsdam Magnetic Model of the Earth (POMME), Geochem. Geophys. Geosyst., 7, Q07008, doi:10.1029/2006GC001269.

Maus, S., H. Lühr, M. Rother, K. Hemant, G. Balasis, P. Ritter and C. Stolle (2007), Fifth generation lithospheric magnetic field model from CHAMP satellite measurements, http://www.gfzpotsdam.de/pb2/pb23/index.html.

Nakagawa, I., T. Yukutake, and N. Fukushima (1985), Extraction of magnetic anomalies of crustal origin from Magsat data over the area of Japanese Islands, J. Geophys. Res, 90(B3), 2609-2615

Olsen, N., H. Lühr, T. J. Sabaka, M. Mandea, M. Rother, L. Tøffner-Clausen, and S. Choi (2006), CHAOS - A Model of Earth 's Magnetic Field derived from CHAMP, Ørsted, and SAC-C magnetic satellite data, Geophys. J. Int., 166, 67-75, doi:10.1111/j.1365-246X.2006.02959.x.

Qureshy, M. N. (1982), Geophysical and Landsat lineament mapping-an approach illustrated from West Central and South India, Photogrammetria, 37, 161-184.

Ravat, T., Hildenbrand and W. Roest (2003), New way of forecasting near-surface 
Magnetic data: The utility of the comprehensive model of the magnetic field, The leading edge, 22, 784-785.

Reeves, C.V., and De Wit, M. (2000), Making ends meet in Gondwana: retracing the transforms of the Indian Ocean and reconnecting continental shear zones, Terra Nova, 12, 272-280, doi:10.1046/j.1365-3121.2000.00309.x.

Sabaka, T. J., N. Olsen, and M. Purucker (2004), Extending comprehensive models of the Earth's magnetic field with Ørsted and CHAMP data, Geophys. J. Int., 159(2), 521-547,

Snyder, J.P (1987), Map projections - A working manual, US Geological Survey,

Professional paper 1395.

Thomson, A., and V. Lesur (2006), An improved geomagnetic data selection algorithm for global geomagnetic field modelling, Geophys. J. Int., In press.

Vine, F. J. (1966), Spreading of the Ocean Floor: New Evidence, Science, 154, 3775, 1405-1515.

Wessel, P., and W. H. F. Smith (1991), Free software helps map and display data, Eos Trans., AGU, 72, 441. 
Table 1 Data used in this study and available metadata information.

\begin{tabular}{|c|c|c|c|c|c|}
\hline Compilation name & height $(\mathrm{m})$ & Coordinate system/projection & Reduction & References & Index \\
\hline Antarctic & N/A & Geographic & $\mathrm{N} / \mathrm{A}$ & ADMAP, http://mww.geology.ohio-state.edu/geophys/admap/ & 47 \\
\hline Arctic compilation & 1000 & Geographic & Filtered $500 \mathrm{~km}$ & Voreof & 41 \\
\hline Europe & 3000 & Lambert conic conforme 30/60-20 & DGRF1980 & Wonik et al., 2001 & 17 \\
\hline France & 3000 & LLambert II etendu & Total field 1964.5 & $\begin{array}{l}\text { Le Mouel, } 1969 \\
\end{array}$ & 12 \\
\hline China & & Geographic & $\mathrm{N} / \mathrm{A}$ & N/A & \\
\hline Middle East & 1000 & Geographic & $\mathrm{N} / \mathrm{A}$ & AAIME, http://home.casema.nl/errenwijlens/itc/aaime/ & 31 \\
\hline Australia & 1000 & $\mathrm{~N} / \mathrm{A}$ & $\mathrm{N} / \mathrm{A}$ & Geoscience Australia, http:///mww.ga.gov.au/ & 61 \\
\hline USSR & 500 & Geographic & VSEGEI 1965 & N/A & \\
\hline Russia & N/A & N/A & N/A & VSEGEl, http://www.vsegei.ru/WAY/247038/locale/EN/ & 23 \\
\hline South East Asia & & Geographic & $\mathrm{N} / \mathrm{A}$ & CCOP, http:///www.ccop.or.th & 37 \\
\hline North America & varying & NAD27 & CM4 varying & NAMAG, http://pubs.usgs.gov/sm/map map/ & 43 \\
\hline South Africa (SaNaBoZ) & 1000 & Geographic & N/A & SADC, http://www.sadc.fi & 71 \\
\hline ttaly & & Geographic & ItGRF 1979 & Chiappini, C., Annali di Geofisica, Vol 45, 5, 2000 & \\
\hline Finland & 150 & WGS84 & DGRF-65 & GTK, http:///mww.gtk.fi & \\
\hline Fennoscandia & & WGS84 & DGRF-65 & GTK, http://Www.gtk.fi & 11 \\
\hline Spain & 3000 & OTM33N & GRF1987 & Socias, l., Earth Planet Sci Lett, 105, 55-64, 1991 & \\
\hline Canary Island & 3200 & OTM $28 \mathrm{~N}$ & IGRF 1993.79 & \begin{tabular}{|l|} 
IGN, Publi. Tec., No35, Madrid, 1996 \\
\end{tabular} & 67 \\
\hline Eurasia & 5000 & Geographic & $\mathrm{N} / \mathrm{A}$ & GSC, http://gsc.nrcan.gc.ca & 29 \\
\hline \begin{tabular}{|l|l|l} 
Austria \\
\end{tabular} & 5000 & GK, M31 & $\mathrm{N} / \mathrm{A}$ & GSA, http://www.geologie.ac.at & 19 \\
\hline Marine track-lines & varying & Geographic & CM4 varying & NGDC, http://www.ngdc.noaa.gov/mgg/geodas/trackline.html & 83 \\
\hline Africa and south america & 5000 & Geographic & Varying IGRF & GETECH, http:///www.getech.com & 89 \\
\hline India & 5000 & Geographic & N/A & Qreschy, M.N., 1982, Photogrammetria, 37, 161-184. & 73 \\
\hline India & 5000 & Geographic & N/A & GSI, http:///Www.gsi.gov.in & 79 \\
\hline \begin{tabular}{|l|l} 
Project Magnet \\
\end{tabular} & 4750 & geographic & Comprehensive models & NGDC, http://Www.ngdc.noaa.gov/seg/geomag/proj mag.shtml & \\
\hline Argentina inland & 5000 & N/A & N/A & SEGEMAR,http://www.segemar/gov.ar & 53 \\
\hline Argentina margin & 5000 & Geographic & $\mathrm{N} / \mathrm{A}$ & Ghidella, DNA, http://www.dna.gov.ar & 59 \\
\hline MF5 & 5000 & geographic & POMME 3.1 & http://www.gfz-potsdam de/pb2/pb23/index.html. & 97 \\
\hline
\end{tabular}


Table 2: Original statistics. From left to right: Grid name, number of points, minimum and maximum anomaly intensity, standard deviation, and arithmetic sum.

\begin{tabular}{|c|c|c|c|c|c|c|}
\hline Grid Name & Grid size & $\delta \mathrm{Fmin}$ & $\delta \mathrm{Fmax}$ & $\langle\delta \mathrm{F}\rangle$ & $\sigma$ & $\Sigma$ \\
\hline Africa and South America & 65030 & -1058 & 633 & $3 . E+00$ & 66 & 86615 \\
\hline Antarctica & 969479 & -924 & 2140 & 9.E-01 & 124 & 823531 \\
\hline Arctic & 2225311 & -2573 & 5504 & $-2 . E-01$ & 167 & -514358 \\
\hline Argentina continent & 571585 & -3225 & 1416 & 5.E-01 & 108 & 271092 \\
\hline Argentina margin & 44092 & -146 & 313 & $-7 . E+00$ & 46 & -326009 \\
\hline Australia & 23636551 & -8786 & 18823 & $-7 . E+00$ & 195 & -171626308 \\
\hline Austria & 4240 & -36 & 147 & 1.E+01 & 26 & 53838 \\
\hline Canary & 328077 & -309 & 545 & $9 . E+01$ & 95 & 28420981 \\
\hline East India & 625898 & -4018 & 7076 & $5 . E+00$ & 102 & 3272122 \\
\hline Eurasia & 1758876 & -879 & 1066 & $-5 . E-01$ & 120 & -803297 \\
\hline Europe & 664626 & -1689 & 8035 & $2 . E+01$ & 180 & 10710206 \\
\hline Fennoscandia & 78861 & -498 & 1307 & 1.E+01 & 160 & 896232 \\
\hline Finland & 420121 & -2111 & 4005 & 2.E+00 & 241 & 821084 \\
\hline France & 7560 & -105 & 254 & $3 . E+00$ & 29 & 19712 \\
\hline Italy & 36603 & -732 & 1472 & $1 . E+01$ & 79 & 430837 \\
\hline Magnet Project & 7814111 & -9571 & 22527 & $-2 . E+01$ & 94 & -149868984 \\
\hline Marine Data & 2379244 & -25809 & 45932 & $-2 . E+00$ & 930 & -57049801 \\
\hline Mexico & 79699 & -723 & 718 & $-2 . E+02$ & 86 & -12962119 \\
\hline Middle East & 4915299 & -5465 & 2413 & $-1 . E+00$ & 97 & -4869716 \\
\hline NAMAG & 63447385 & -22724 & 27540 & 5.E-01 & 192 & 34263569 \\
\hline Russia & 1061053 & -978 & 9605 & $6 . E+01$ & 350 & 58444363 \\
\hline Sanabozi & 12324191 & -14583 & 11433 & $6 . E+01$ & 176 & 43554434 \\
\hline South Asia & 3554249 & -990 & 2197 & $-3 . E+01$ & 84 & -97825986 \\
\hline Spain & 81501 & -86 & 251 & $1 . E+01$ & 21 & 1100325 \\
\hline USSR & 14481793 & -32685 & 32560 & 4.E+01 & 253 & 578134196 \\
\hline
\end{tabular}


Table 3: Statistics after pre-processing and outliers correction. Same caption as Table 2.

\begin{tabular}{|c|c|c|c|c|c|c|}
\hline Grid Name & Grid size & $\delta \mathrm{Fmin}$ & $\delta \mathrm{Fmax}$ & $\langle\delta \mathrm{F}\rangle$ & $\sigma$ & $\Sigma$ \\
\hline Africa and South America & 65030 & -1032 & 701 & $13-10$ & 66 & 3.E-08 \\
\hline Antarctica & 969479 & -894 & 2113 & 6.E-11 & 128 & $6 . E-05$ \\
\hline Arctic & 2225311 & -2604 & 5528 & $-2 . E-11$ & 171 & $-5 . E-05$ \\
\hline Argentina continent & 571585 & -3150 & 1480 & $4 . E+04$ & 107 & $-1 . E-07$ \\
\hline Argentina margin & 44056 & -137 & 318 & 1.E-13 & 44 & $6 . E-09$ \\
\hline Australia & 23636550 & -8743 & 18882 & 3.E-11 & 209 & 8.E-04 \\
\hline Austria & 4240 & -55 & 137 & 3.E-15 & 27 & $0 . E+00$ \\
\hline Canary & 328077 & -400 & 456 & 3.E-11 & 87 & 1.E-05 \\
\hline East India & 625898 & -4001 & 7109 & 1.E-11 & 102 & 8.E-06 \\
\hline Eurasia & 1758876 & -916 & 1034 & $-2 . E-11$ & 123 & 4.E-05 \\
\hline Europe & 664626 & -1712 & 7983 & $-2 . E-11$ & 179 & 1.E-06 \\
\hline Fennoscandia & 78862 & -526 & 1254 & $-7 . E-13$ & 158 & $6 . E-09$ \\
\hline Finland & 420121 & -2110 & 3974 & $6 . E-11$ & 240 & 2.E-05 \\
\hline France & 7560 & -111 & 251 & 3.E-15 & 29 & $0 . E+00$ \\
\hline Italy & 36603 & -747 & 1402 & $6 . E-14$ & 79 & 2.E-09 \\
\hline Magnet Project & 7814111 & -2000 & 2000 & $-3 . E-07$ & 89 & $0 . E+00$ \\
\hline Marine Data & 19455835 & -2000 & 2000 & $-6 . E-12$ & 148 & 1.E-04 \\
\hline Mexico & 79699 & -562 & 876 & 2.E-12 & 86 & $-1 . E-07$ \\
\hline Middle East & 4915299 & -5446 & 2408 & 1.E-111 & 101 & 5.E-04 \\
\hline NAMAG & 63447385 & -22567 & 26232 & 4.E-10 & 191 & $3 . E-02$ \\
\hline Russia & 1061053 & -1046 & 9545 & $-4 . E-11$ & 349 & 5.E-05 \\
\hline Sanabozi & 12324191 & -14642 & 11341 & $-8 . E-12$ & 176 & $-1 . E-04$ \\
\hline South Asia & 3554249 & -340 & 2196 & 2.E-11 & 85 & 6.E-05 \\
\hline Spain & 81501 & -97 & 242 & 7.E-14 & 20 & $0 . E+00$ \\
\hline USSR & 14481793 & -32138 & 31455 & $5 . \mathrm{E}-10$ & 249 & 8.E-03 \\
\hline
\end{tabular}




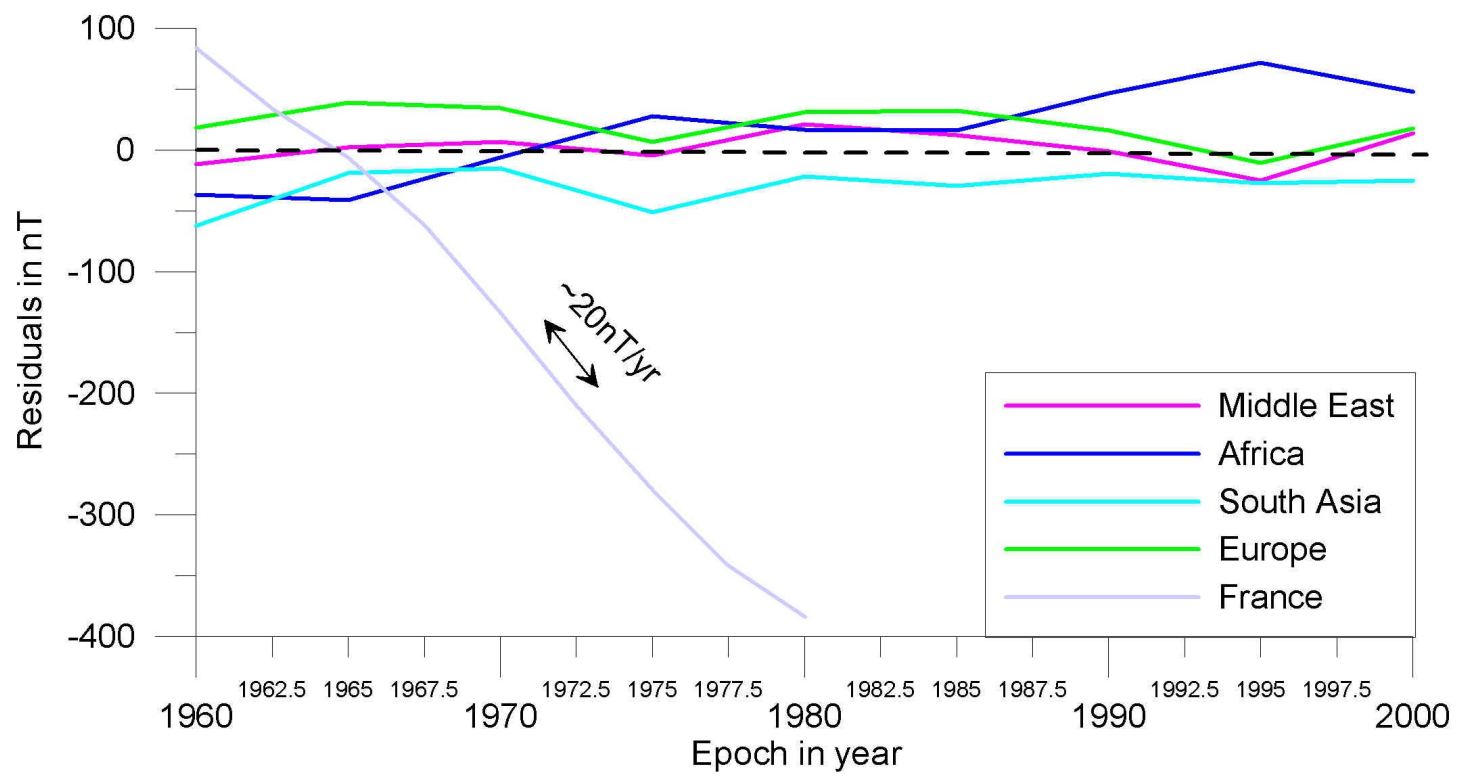

Figure 1: Compilations and individual surveys are iteratively corrected from main field using DGRF and CM4 for each period. The reduction epoch is selected for the minimum anomaly field. 

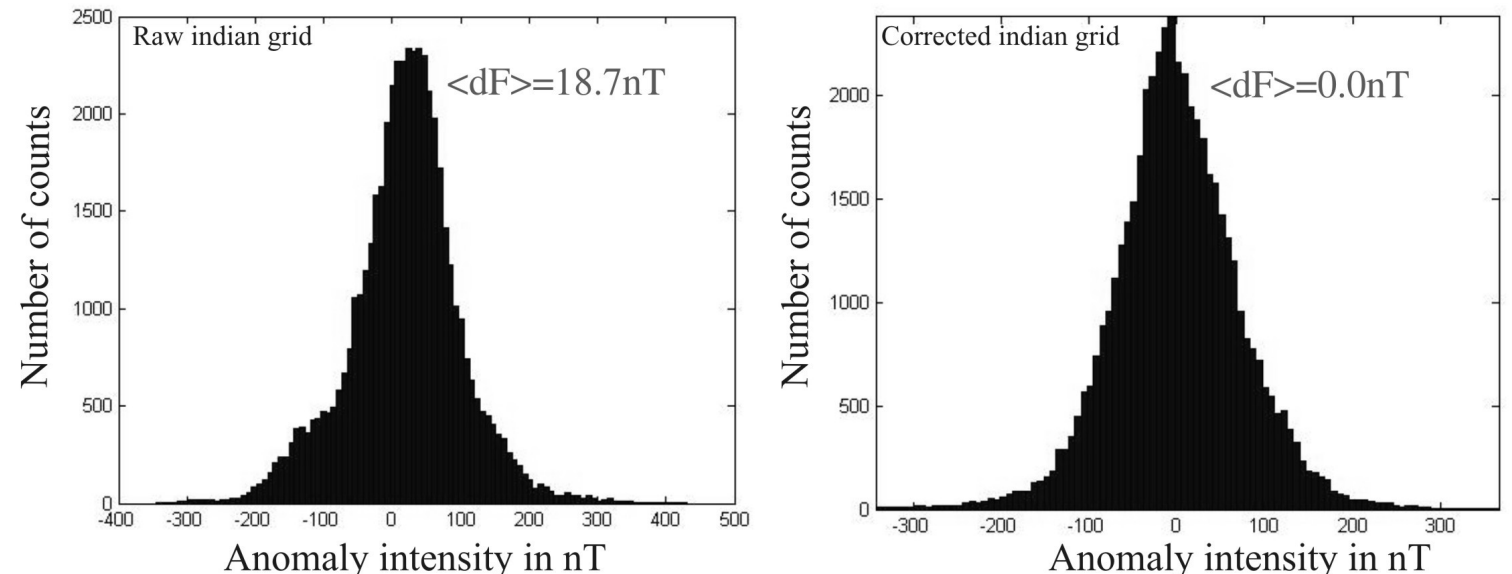

Figure 2: Example of an anomaly intensity distribution before and after main field and polynomial corrections for the East Indian grid. 

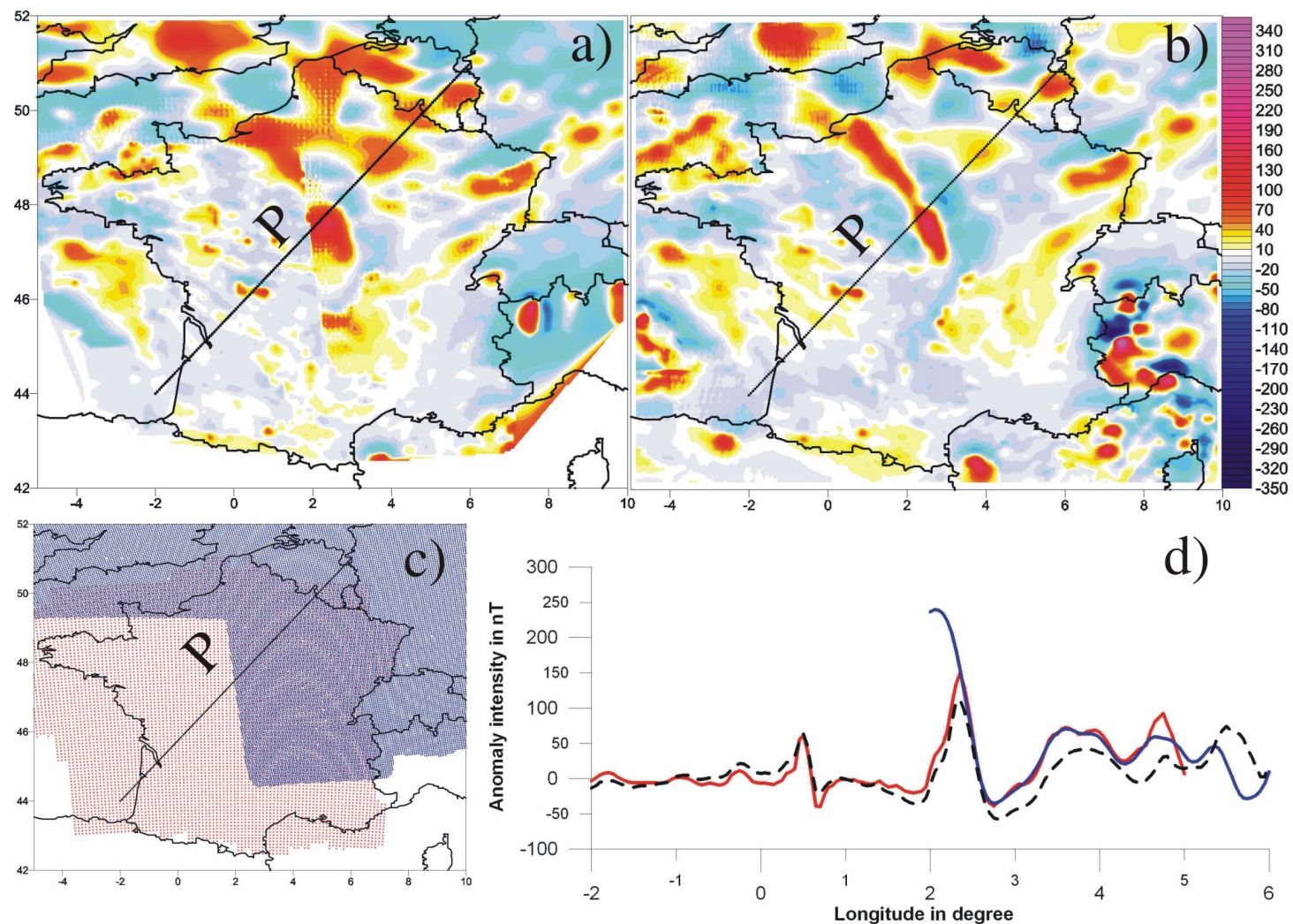

Figure 3: Overlapping area before a) and after b) processing over Western Europe. Data distributions for the European (blue) and the French (red) grids are shown in $c$ ). Profiles $P$ are sketched in d). In blue the European compilation, in red the French grid and in black the profile over the map at its final stage. 


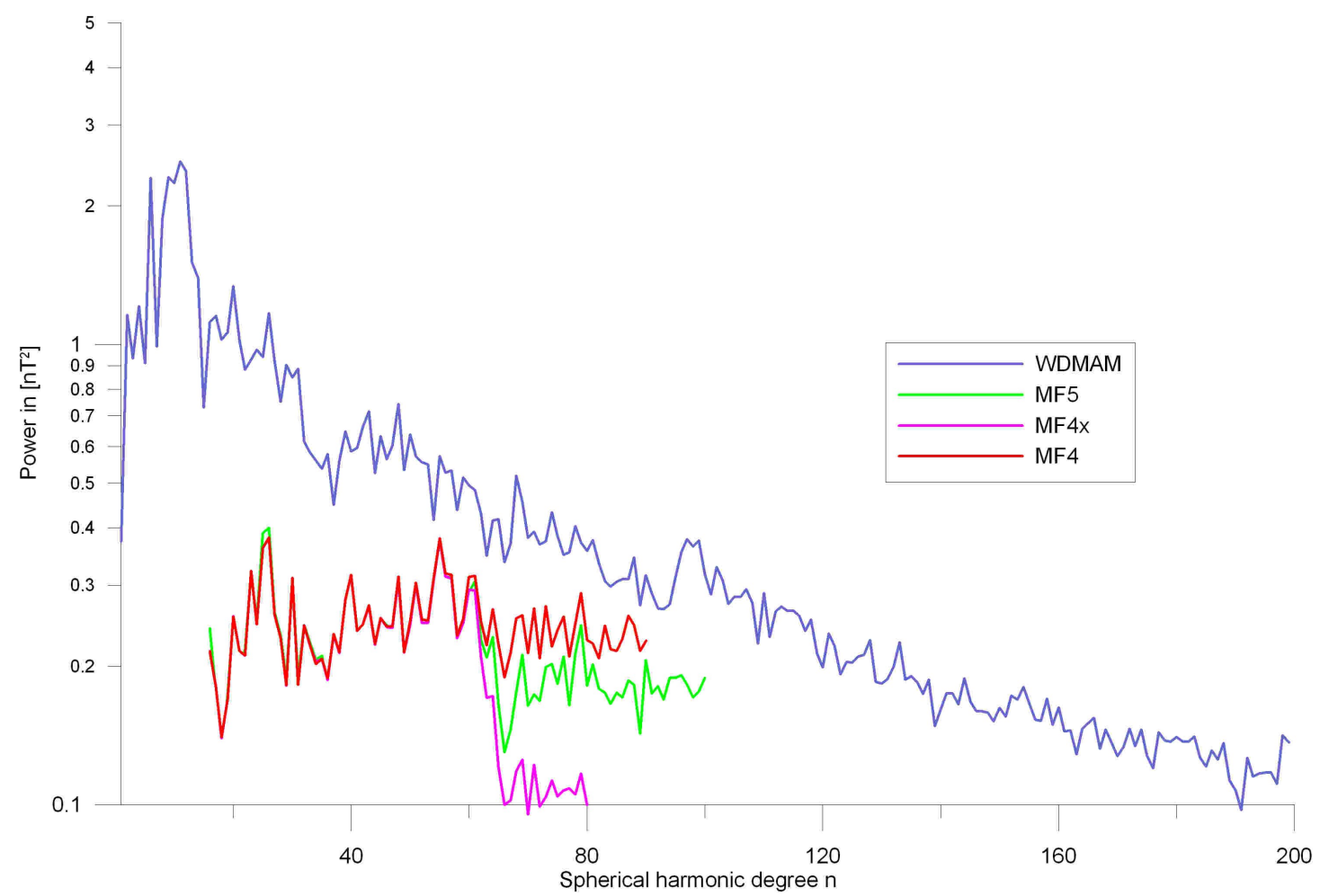

Figure 4: Spectra of the near-surface data and satellite based models. A small increase around degree 90 suggests that some data were reduced with a global field model. 


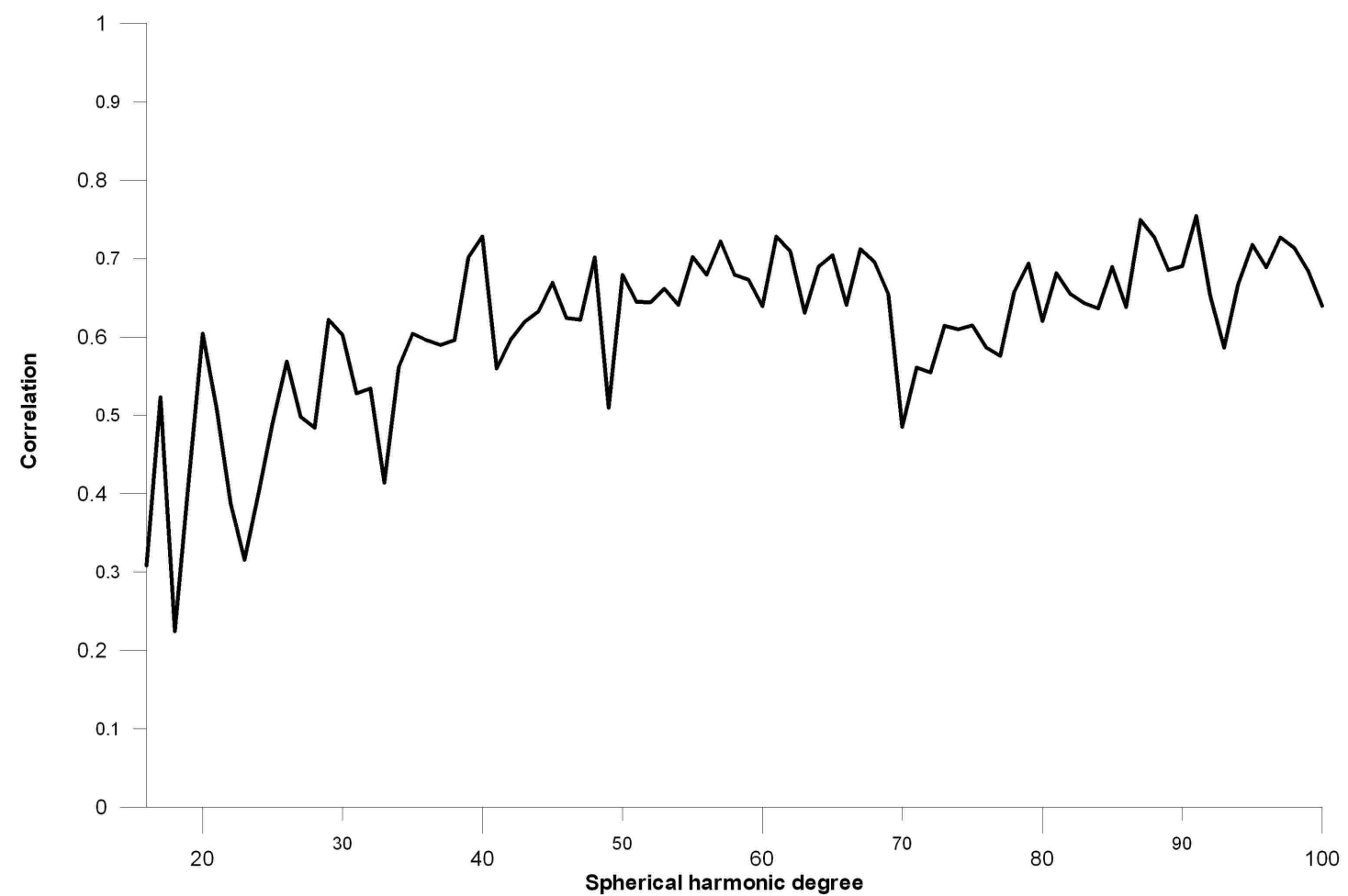

Figure 5: Spherical Harmonic Correlation Analysis between ground based and satellite models shows a stable correlation from degree 40. 
Figure 6: Our candidate WDMAM model in a Mollweide projection (see file attached). 